\title{
A distributed transducer system for functional electrical stimulation
}

\author{
Gudnason, Gunnar; Nielsen, Jannik Hammel; Bruun, Erik; Haugland, Morten
}

Published in:

Proceedings on 8th IEEE International Conference on Electronics, Circuits and Systems

Link to article, DOI:

10.1109/ICECS.2001.957763

Publication date:

2001

Document Version

Publisher's PDF, also known as Version of record

Link back to DTU Orbit

Citation (APA):

Gudnason, G., Nielsen, J. H., Bruun, E., \& Haugland, M. (2001). A distributed transducer system for functional electrical stimulation. In Proceedings on 8th IEEE International Conference on Electronics, Circuits and Systems (Vol. 1) https://doi.org/10.1109/ICECS.2001.957763

\section{General rights}

Copyright and moral rights for the publications made accessible in the public portal are retained by the authors and/or other copyright owners and it is a condition of accessing publications that users recognise and abide by the legal requirements associated with these rights.

- Users may download and print one copy of any publication from the public portal for the purpose of private study or research.

- You may not further distribute the material or use it for any profit-making activity or commercial gain

- You may freely distribute the URL identifying the publication in the public portal 


\title{
A distributed transducer system for functional electrical stimulation
}

\author{
Gunnar Gudnason, Jannik H. Nielsen and Erik Bruun \\ Ørsted-DTU, Technical University of Denmark, DK-2800 Lyngby \\ gg@oersted.dtu.dk \\ Morten Haugland \\ Center for Sensory-Motor Interaction, Aalborg University, DK-9220 Aalborg
}

\begin{abstract}
Implanted transducers for functional electrical stimulation (FES) powered by inductive links are subject to conflicting requirements arising from low link efficiency, a low power budget and the need for protection of the weak signals against strong $R F$ electromagnetic fields.

We propose a solution to these problems by partitioning the $R F$ transceiver and sensor/actuator functions onto separate integrated circuits. By amplifying measured neural signals directly at the measurements site and converting them into the digital domain before passing them to the transceiver, the signal integrity is less likely to be affected by the inductive link. Neural stimulators are affected to a lesser degree, but still benefit from the partitioning.

As a test case, we have designed a transceiver and a sensor chip which implement this partitioning policy. The transceiver is designed to operate in the $6.78 \mathrm{MHz} I S M$ band and consumes approximately $360 \mu \mathrm{W}$. Both chips were implemented in a standard $0.5 \mu \mathrm{m}$ CMOS technology, and use a $3 \mathrm{~V}$ supply voltage.
\end{abstract}

\section{Introduction}

The subject of this article is a solution for several problems which plague designers of inductively powered biomedical implants for functional electrical stimulation. Inductive links are commonly employed to power and control such implants since they provide a wireless connection, which is desirable since percutaneous wires provide an infection path into the body. Inductive links also eliminate the need for an implanted battery, which might eventually need to be replaced. The price which must be paid for these advantages is the relatively high power dissipation in the external (non-implanted) apparatus due to the low efficiency of the link, and a degradation of the signals under examination, because of the high electromagnetic fields. While the RF signals normally lie far outside the biological signal band, they can easily desensitize an amplifier designed for a $10-20 \mu \mathrm{V}$ signal range.

The signal amplitude quoted above is typical for signals obtained using cuff electrodes [1]. Stimulators connected to cuff electrodes generate much larger signals, so they are not affected to the same extent as sensors by the external RF field.

We propose therefore a physical partitioning of the signal processing functions, in order to limit the transmission

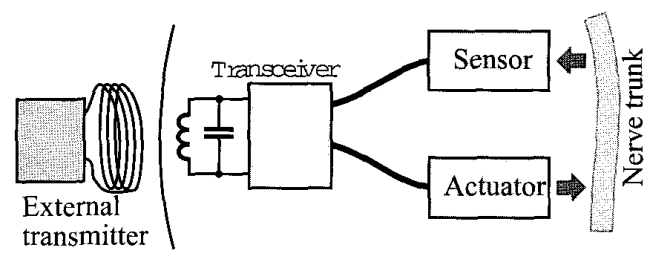

Figure 1. A partitioning example for a FES system containing a transceiver chip and sensor and actuator chips. The last two are not necessarily connected to the same nerve trunk.

distances for sensitive signals, and to allow optimal placement of critical functions of the system. The conflicting design criteria which can be accommodated to a large extent by partitioning are:

- The RF part of the system should be close to the skin surface for better power transmission, and also for better link bandwidth. The proximity may also be an advantage for surgical access.

- The transducers should be placed close to the active sites, which can be relatively deep inside the body.

- Long routing of transducer signals should be avoided, as the strong electromagnetic field will induce an RF interferer overlaid on the desired signal. Physical separation of the transducer from the RF link also reduces the problems, since the field falls off rapidly with distance (as $1 / r^{3}$ in the far-field limit).

- The system must contain a considerable amount of digital logic, for control, timing, and buffering of data. The logic will inevitably couple switching noise onto the supplies and into the substrate. Using low-noise logic solutions like current-steering logic [3] can eliminate the problem, but the static supply current of CSL and other ultra-low noise logic families makes them unsuitable as the system complexity passes a certain point. Placing the sensors on separate chips isolates them from the logic supply noise.

One option which was examined, and which alleviates some of the problems above, is to place only the receiver 


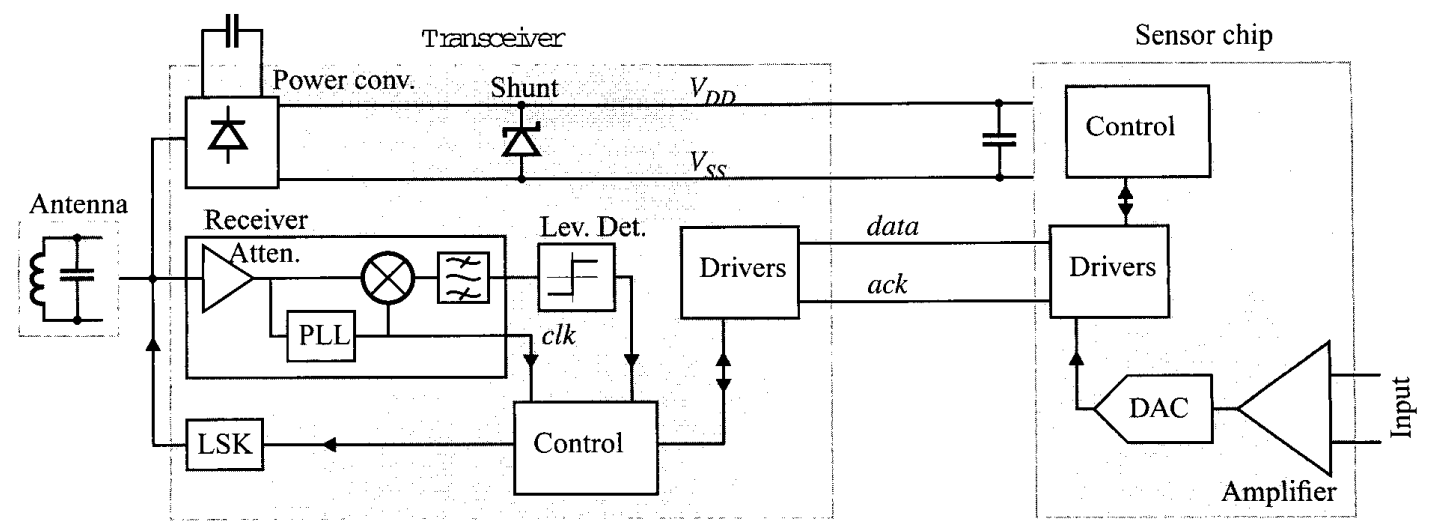

Figure 2. A simplified block diagram of the system, showing the main parts of the transceiver and sensor chips. Differential signals are shown with a single line for clarity.

coil close to the skin surface, and route the antenna signal to a single integrated transceiver/transducer. This is however not practical since biocompatible interconnect solutions like Cooper cable ${ }^{1}$ have a high impedance at DC and RF.

We adopted a solution with simple digital communication protocols between the central transceiver and control chip, and the outlying transducer chips. The protocol was designed so that it could be accommodated along with the power supply wires in a simple 4-wire flexible cable. In this specific test case, we implemented a simple system with one transceiver chip and one sensor chip. The sensor chip includes an amplifier for cuff electrode signals, which is itself the subject of another article [2], an AD converter and bus interface logic. The transceiver includes a direct conversion receiver (actually a homodyne), a load modulation circuit for transmission of data out of the system, control logic, supply regulators and references.

\section{System description}

The following is a description of the transceiver chip and the aspects relating to communication between it and the other parts of the system. Since the main focus of this article is on the way in which partitioning of functions can solve some of the problems in an implanted system of this type, the sensor chip will only be described inasmuch as it relates to the top-level design of the system. The principle of partitioning applies to any transducer type that is relevant in an implanted system, so the internals of the sensor chip will not be emphasized. Figure 2 shows a toplevel diagram of the system.

\subsection{The link}

The use of inductive links for power and data transmission is described in great detail elsewhere, see for example $[5,6]$.

By separating the transceiver chip and the antenna, it is possible to reduce the distance between the external trans-

\footnotetext{
${ }^{1}$ Produced by Finetech Medical Ltd.
}

mitter antenna (a tuned LC circuit) and the internal one to $15-20 \mathrm{~mm}$. The coupling coefficient for normal coil geometries at this distance is on the order of 0.01-0.05. This permits far better power transfer than if the transceiver were placed together with the transducer, and makes load modulation a viable method to extract information from the system. Systems with smaller coupling coefficients must resort to active transmission of signals to the outside [4], which increases the power consumption.

The chosen modulation method is PAM with Manchester encoding and a modulation index of approximately 0.2 . This is compatible with high-efficiency class $\mathrm{D}$ or $\mathrm{E}$ transmitter configurations $[7,8]$. The carrier frequency is $6.78 \mathrm{MHz}$ which coincides with one of the ISM (industrial, scientific and medical) bands. The target bit rate is $50 \mathrm{kbit} / \mathrm{s}$.

\subsection{The power supply}

The power is extracted from the RF carrier by means of a full-wave bridge rectifier, which is implemented by using diode-connected $\mathrm{P}$-channel MOS transistors in a common N-well, as shown in figure 3. The standard CMOS process does not offer high-quality floating diodes, so another solution must be chosen. The available elements are three types of $\mathrm{p}-\mathrm{n}$ junction and diode-connected MOS transistors, but these all suffer from high substrate currents and/or parasitic elements which divert some of the current from its intended path. The PMOS rectifier bridge is accompanied by parasitic vertical PNP transistors which can divert some of the input current to $V_{S S}$ instead of to $V_{D D}$.

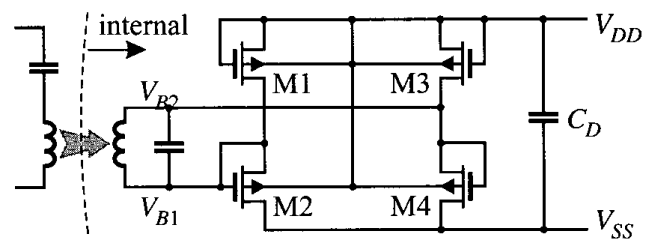

Figure 3. The power conversion circuit. 


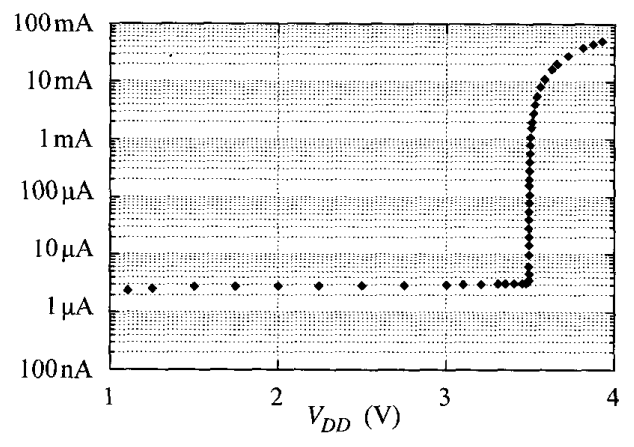

Figure 4. The measured shunt regulator current.

We have solved this problem by dimensioning the PMOS transistors so that they are biased in weak inversion over the entire operating range. Since the nominal threshold voltage is about $0.6 \mathrm{~V}$, there is a range of drain-gate voltages for $\mathrm{M} 1$ in figure 3 (corresponding to the emitter-base potential in the parasitic PNP) where the MOS current dominates the current through the bipolar transistor by a large factor. The current-handling capability of the rectifiers can be increased for a given maximum parasitic/main current ratio, by increasing the width (and area) of the transistors. The main penalty is an increased input capacitance. Our experimental data show that the parasitic current ratio is smaller than 0.005 for a system supply current of $200 \mu \mathrm{A}$ and an input capacitance of $2 \mathrm{pF}$. All the transistors in figure 3 have $W / L=800 / 0.5$ in micrometers.

The transducer chip contains an active shunt regulator which provides protection against excessive input power. The transmitted power is a very strong function of antenna spacing, so an unprotected circuit can easily be burned out. The shunt circuit is basically a pass transistor between $V_{D D}$ and $V_{S S}$, and a feedback loop which compares $V_{D D}$ to a bandgap reference voltage. As the supply voltage approaches the trigger point (which was set equal to the maximum supply voltage for the technology), the regulator begins shunting current from the supply, and the current changes by 4 decades over a short supply voltage interval (see figure 4 ).

The performance of the power supply conditioning system is shown in figure 5. The conversion of the RF power to a DC supply voltage and the overvoltage protection is carried out mostly on-chip, with the only external component being the energy storage capacitor $C_{D}$. The transmitter used in these measurements was running at a relatively high power level, witnessed by the fact that the full supply voltage is reached at a separation of $120 \mathrm{~mm}$. Despite the strong dependence of the magnetic field amplitude on the separation $d$, the on-chip shunt succeeds in maintaining the supply voltage at the nominal level at all separations.

The transceiver chip provides the supply voltage for the other chips in the system. Two strands out of four in the Cooper cable have been assigned to $V_{S S}$ and $V_{D D}$. Because of the helically wound construction of the cable, and the Pt-Ir composition, the impedance is relatively high at all frequencies. To provide a stable supply voltage for the

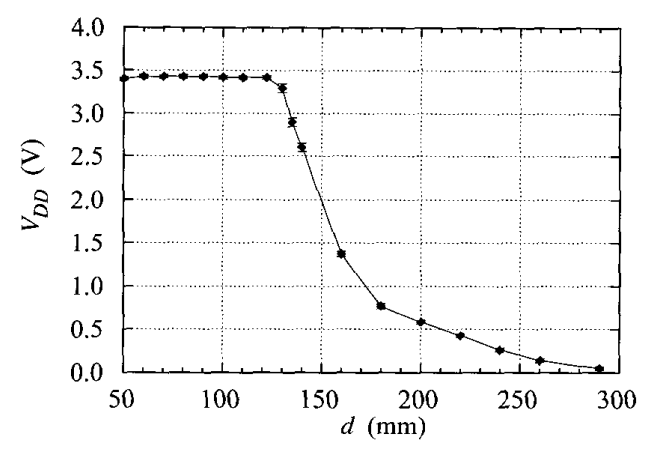

Figure 5. The measured on-chip supply voltage as a function of the separation between the transmitter and transceiver antennas.

outlying chips, it is therefore necessary to add decoupling capacitors to the supply lines at the transducer ends. A stimulator chip can require relatively large current pulses from the supply while delivering a stimulus.

\subsection{Transceiver}

The receiver part of the transceiver chip is a directconversion receiver. Since the input signal has a larger amplitude than the supply range, an attenuator with a gain of approximately 0.1 is inserted in the signal path. The input signal is tracked by a PLL which also provides the system clock (the block diagram does not show a clock divider circuit which divides the clock frequency down to $1.7 \mathrm{MHz}$ )

The output of the PLL and the input signal are put through a mixer, and the mixer output is filtered in a 4thorder differential $G_{m}-C$ filter. The filter output is directed to a mixed analog-digital level detector, which detects the sign of the transmitted data bits. Instead of using a partly analog level detector, the normal processing method would be to sample the filter output for further digital signal processing. This does however require more sophisticated digital circuits than we were willing to implement, and possibly requires more supply current.

The target bit rate for the system was $50 \mathrm{kbit} / \mathrm{s}$, and the receiver is designed to handle up to $100 \mathrm{kbit} / \mathrm{s}$.

Data is transmitted out of the system by load modulation. The reflected impedance seen by the external transmitter is varied by changing the load seen by the secondary LC circuit. A switch is connected between the terminals of the antenna, and by closing the switch, a maximum change in the reflected impedance is obtained. This simple scheme has the disadvantage of stopping power transfer to the system during load modulation, and other load modulation circuits have been designed to avoid this [9]. The duty cycle of the switch closure is however so low in our case that the reduction in average power transfer is small.

\subsection{Interchip communication}

The cable type that we used as a reference for our interconnects is, as mentioned before, a 4-strand biocompati- 
ble cable type by the name of Cooper cable. In addition to being biocompatible, the cable is wound in a helical pattern and embedded in silicone, so it can be stretched. The stretching reduces the probability of damage to the surrounding tissue.

Because of the biological constraints that the cable must fulfill, it does not have very good electrical properties, and the use of the cable must be adjusted accordingly. We measured the series resistance at DC of a representative length of the cable, and found it to be $210 \Omega / \mathrm{m}$. The capacitance between any two wires varies from 20 $80 \mathrm{pF} / \mathrm{m}$ because of the lack of symmetry, and the capacitance of each wire to the surroundings is about $50 \mathrm{pF} / \mathrm{m}$.

No clock signal is sent across the connection between the chips. Instead, we use an asynchronous handshake mechanism to control data transfer, and an internal oscillator in the sensor chip to provide a time base.

The line drivers are class $\mathrm{AB}$ circuits which have a quiescent current consumption of $5 \mu \mathrm{A}$ each, and can slew the line voltages with a $50 \mu \mathrm{A}$ current. This type of driver was chosen instead of faster types because this is more than sufficient for the purpose, and by limiting the slewing currents the supply transients are reduced. The high and low voltages on the signal lines are $1.0 \mathrm{~V}$ and $0.3 \mathrm{~V}$ respectively instead of the full supply range, again to reduce supply transients and power consumption.

\section{Measurement results}

Most of the basic functions of the transceiver and sensor chips have been measured, and they behave according to specifications.

The results for the power conversion circuit show that the use of PMOS transistors in weak inversion as rectifiers is an ideal solution for pure CMOS technologies. Current throught the substrate are eliminated by placing everything in an $\mathrm{N}$-well, and the effect of parasitic bipolars are all but eliminated.

The active shunt regulator and other regulators and references on the chip are up to the design criteria. Specifically, the shunt regulator consumes negligible supply current within the normal voltage range, with a very sharp rise in current as the trigger point is exceeded. Previous systems use passive zener-based regulators, whose gradual I-V characteristic provides insufficient overvoltage protection for low-voltage CMOS technologies.

The data link to the transceiver chip was tested by using a Class D transmitter driving the inductive link, with a data rate of $50 \mathrm{kbit} / \mathrm{s}$ and $20 \%$ ASK modulation and Manchester encoding. The data transfer functions according to the specifications, and higher data rates can easily be supported with minor modifications.

\section{Conclusion}

We have demonstrated a partitioning scheme for implanted sensor and actuator devices which places the signal processing functions where they are needed. By using this scheme, it is possible to provide better isolation of weak biological signals from strong external disturbances, while simultaneously reducing the overall power

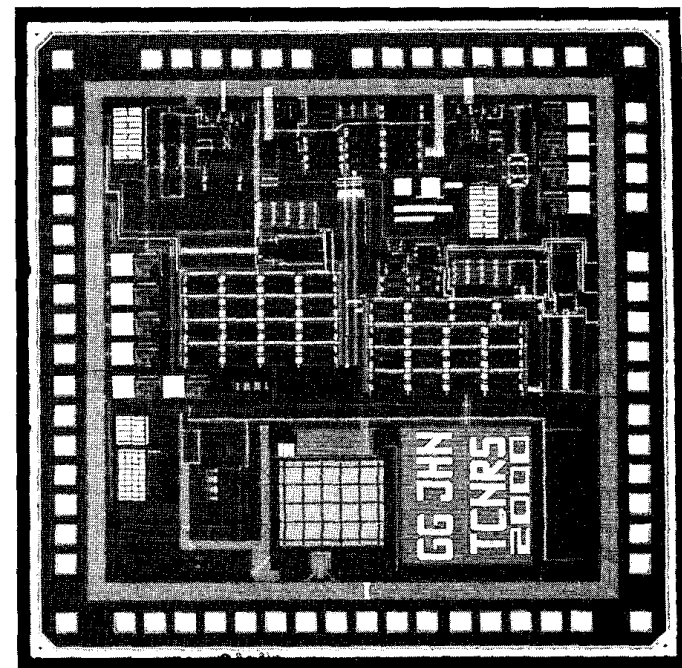

Figure 6. Chip die microphotograph.

consumption by placing the transceiver closer to the external interface. The communication between separate parts of the system has been adapted to existing biocompatible interconnect methods.

We have implemented a simple system consisting of a transceiver and a single sensor chip, but the concept can easily be extended to more general combinations of sensors and actuators, in order to created a complete neural stimulation system with a closed feedback loop.

[1] G. E. Loeb and R. A. Peck, "Cuff electrodes for chronic stimulation and recording of peripheral nerve activity". $J$. Neuroscience Methods, 64 (1996), 95-103.

[2] J. H. Nielsen and T. Lehmann, "An implantable CMOS amplifier for nerve signals", Proc. ICECS 2001.

[3] H.-T. Ng and D. J. Allstot, "CMOS current-steering logic for low-voltage mixed-signal integrated circuits", IEEE Trans. VLSI, 5 (1997), pp. 301-308.

[4] M. Nardin and K. Najafi "A multichannel neuromuscular microstimulator with bi-directional telemetry", 1995 Conf. on Solid-State Sensors and Actuators, pp. 59-62.

[5] D. Galbraith, M. Soma and R. White, "Wide-band efficient inductive transdermal power and data link with coupling insensitive gain", IEEE Trans. BME, 34 (1987), pp. 265275.

[6] N. de N. Donaldson and T. A. Perkins. "Analysis of resonant coupled coils in the design of radio frequency transcutaneous links". Med. and Bio. Eng. and Computing, 21 (1983), pp. 612-627.

[7] A. Djemouai, M. Sawan and M. Slamani, "An efficient RF power transfer and bidirectional data transmission to implantable electronic devices", Proc. ISCAS '99, pp. II-259.

[8] P. R. Troyk and M. A. K. Schwan, "Closed-loop class E transcutaneous power and data link for microimplants" Trans. BME, 39 (1992), pp. 589-599.

[9] Z. Tang et al. "Data transmission from an implantable biotelemeter by load-shift keying using a circuit configuration modulator". IEEE Trans. BME, 42 (1995), pp. 524. 